\title{
Genetic diversity and heterosis prediction of nine sheep populations based on microsatellite markers
}

\author{
Qing Xia ${ }^{1}$, Xiang-Yu Wang ${ }^{1}$, Zhang-Yuan Pan ${ }^{1}$, Ren-Sen Zhang ${ }^{1}$, Ming-Xing Chu ${ }^{1}$, and \\ Ran $\mathrm{Di}^{1}$ \\ ${ }^{1}$ Institute of Animal Science, Chinese Academy of Agricultural Sciences (CAAS)
}

June 4, 2020

\begin{abstract}
The objective of this study was to assess the genetic diversity and population structure of nine sheep populations (four famous mutton breeds worldwide, three popular mutton populations and two most famous high prolific breeds in China) and predict the heterosis among these populations based on microsatellite markers. Results showed the relatively rich genetic diversity in Chinese sheep populations. The results of UPGMA phylogenetic tree and Structure analysis indicated that the nine investigated populations can be divided into two groups. Suffolk and Dorset were clustered in one group, and the other group can be further divided into three clusters: GMM-BAS-BAM, HUS-STH and DOS-DOP. This is consistent with sheep breeding history. TreeMix analysis also hinted the possible gene flow from GMM to SUF. Additionally, based on the calculation of genetic distance, the optimal cross combinations are recommended for two popular mutton sheep populations (BAM and BAS) in the southern Mongolian Plateau and the most famous high prolific breeds (Small Tail Han sheep and Hu sheep) in China, respectively. Together, these results bring us an in-depth view of genetic diversity within sheep population and the genetic relationship among populations.
\end{abstract}

\section{Hosted file}

manuscript.doc available at https://authorea.com/users/329815/articles/456718-geneticdiversity-and-heterosis-prediction-of-nine-sheep-populations-based-on-microsatellitemarkers 

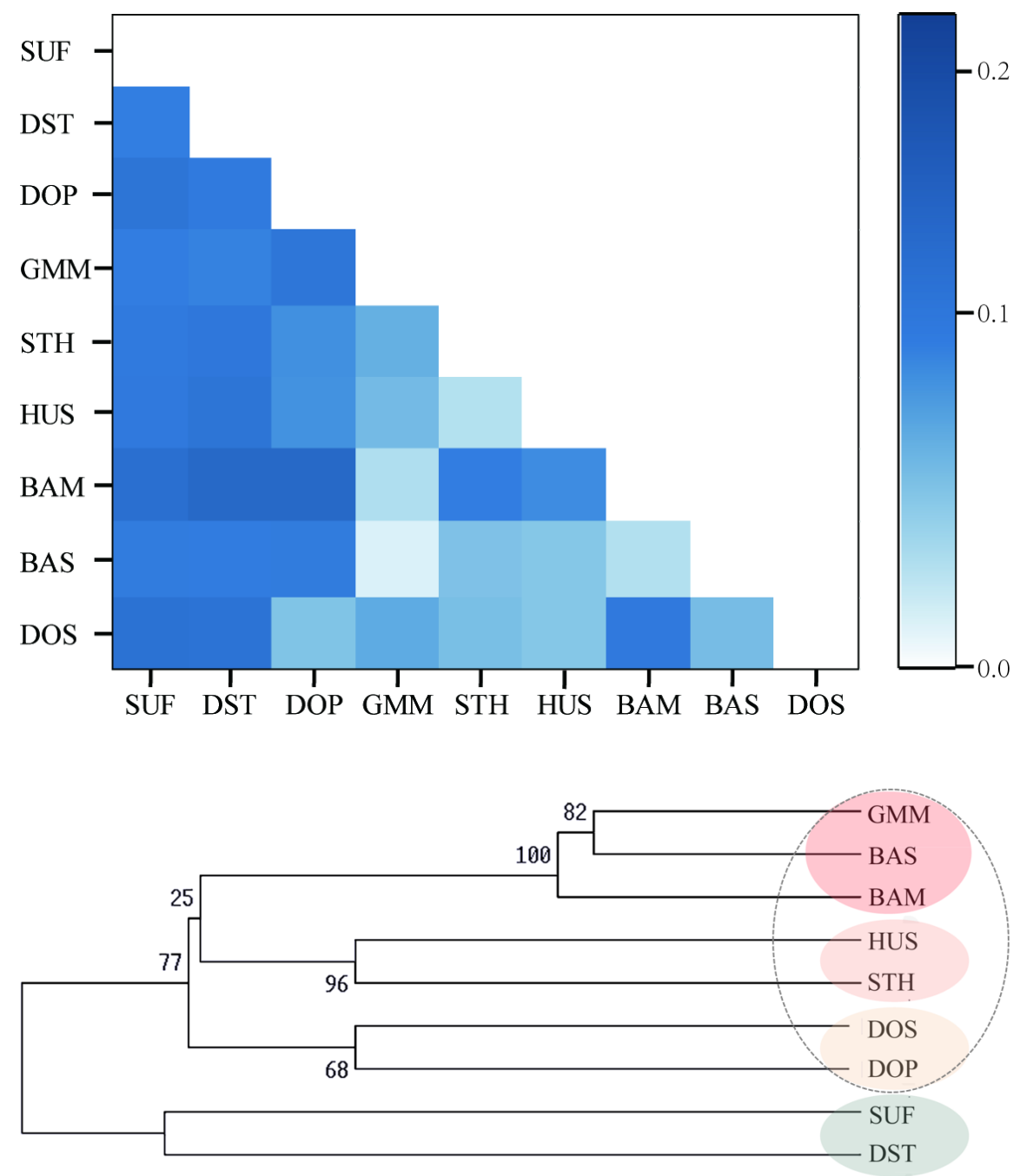

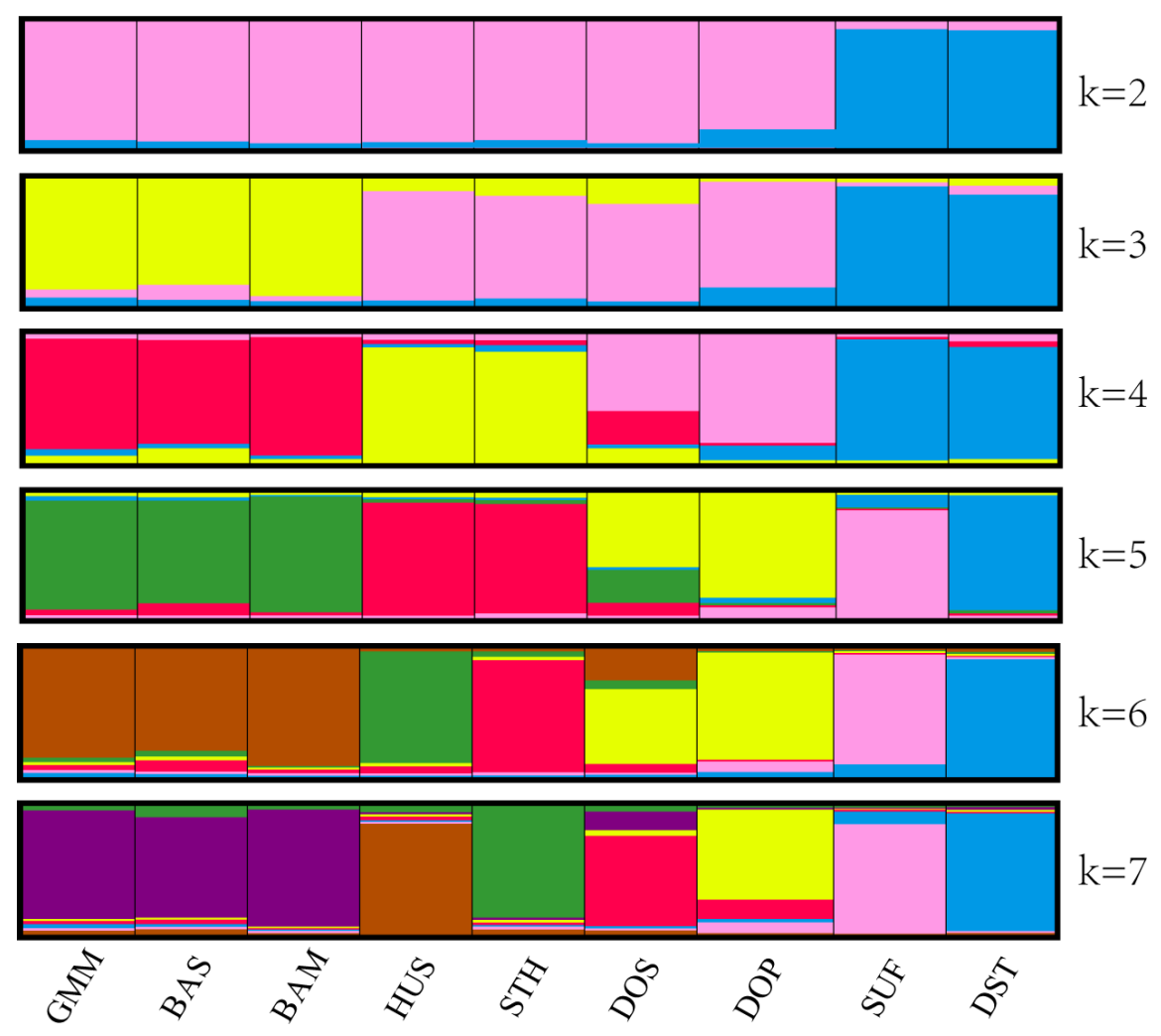
(a)

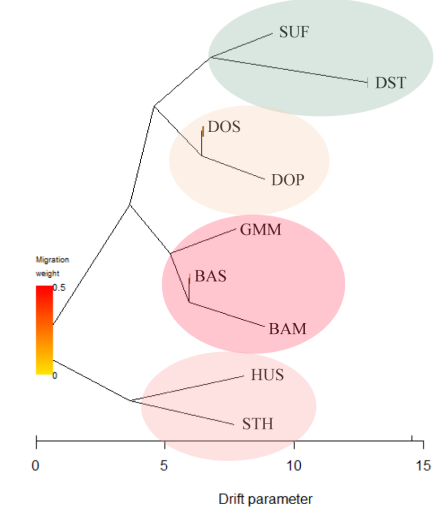

(c)

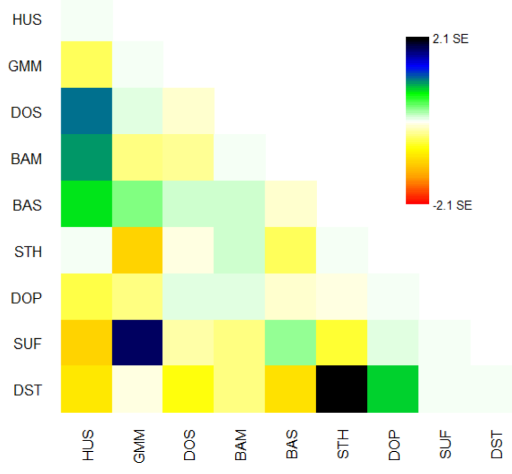

(b)

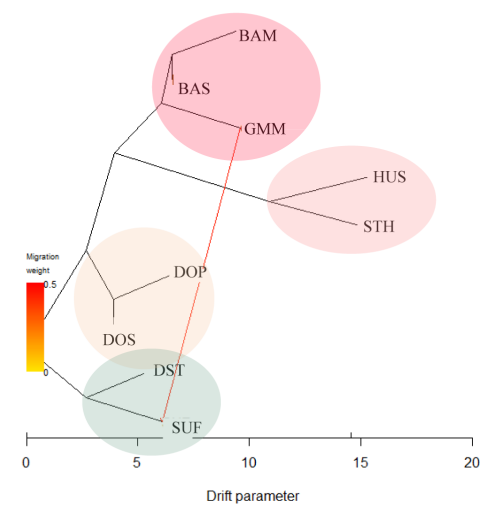

(d)

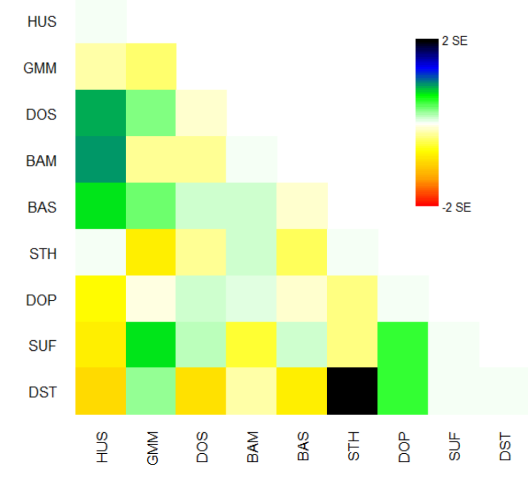

